\title{
It All Starts with a Good Idea: A New Coding System for Analyzing Idea Finding Interactions (AIFI)
}

\author{
Paul C. Endrejat \\ Technische Universität \\ Braunschweig, Germany \\ p.endrejat@tu-bs.de
}

\author{
Annika L. Meinecke \\ Universität Hamburg, Germany \\ annika.luisa.meinecke@uni- \\ hamburg.de
}

\author{
Simone Kauffeld \\ Technische Universität \\ Braunschweig, Germany \\ s.kauffeld@tu-bs.de
}

\begin{abstract}
In today's fast-changing world, teams need to develop a sound capacity for finding new ideas. However, we know little about the behavioral microdynamics that are at the core of creativity in teams. To overcome these shortcomings, we present a new behavioral coding system for analyzing idea finding interactions (AIFI). The AIFI system aims to help researchers study fine-grained creative team processes. In terms of practical application, the AIFI system can serve to visualize the patterns of Idea finding over time. The codes of the AIFI system were derived both inductively (analyzing videos of innovation teams) and deductively (consulting existing coding systems). A first application of the AIFI system showed moderate agreement among coders, speaking to its interrater reliability. Further, we examined distinct relationships between the codes of the AIFI system and (1) ratings of idea quality provided by external raters and (2) team members' perceived effectiveness.
\end{abstract}

\section{Introduction}

Due to an increasingly complex and competitive environment, organizations need to be more adaptable than ever to quickly respond to external challenges. Since tasks become more interconnected, most of these challenges cannot be solved by a single individual but require that teams, ideally consisting of members with diverse disciplinary backgrounds, come together and pull their efforts to drive organizational innovation [29]. As a result, team creativity and team innovation are on the agenda for most organizations and sparked a growing amount of academic research [42]. These efforts cumulated in a growing consensus that we need to gain a better understanding of the creative processes unfolding in teams [8].

It has long been acknowledged that we cannot simply put gifted individuals together in a group and expect creative results [38]. One important framework in this line of research is McGrath's [23] inputprocess-output model that emphasizes the central role of team processes as the core mechanism by which team members combine their individual resources, such as their creative skills, to master team task demands and generate (creative) output [16].

Focusing on (creative) team processes requires a temporal perspective that is sensitive to the ebbs and flows of idea generation over time. To tackle this temporal perspective and to understand how team interaction shapes team outcomes, more and more team process research makes use of observational techniques and specifically behavioral coding $[12,26$, 34]. "Coding" in this context describes that behavioral units (e.g. a sentence that comes up during a group discussion) will be assigned to a behavioral category (e.g. the code "new idea" or "question"). That way, researchers can trace the moment-to-moment dynamics of team interactions which is pivotal to unravel the underlying behavioral mechanisms that lead to more or less successful team interactions [1].

A focus on fine-grained team processes is especially important for innovation teams whose members typically have a diverse disciplinary background. Due to different mindsets and working modes, managing interdisciplinary teams is challenging [29]. Moreover, teams-compared to individuals working alone - tend to create extreme ideas, either in a positive way towards the best outcome, or in a negative way towards the worst outcome [40]. Thus, there is need to understand how the forces that are at play during creative problemsolving can be channeled in a desirable direction, leading to novel and useful ideas $[9,21]$.

As a result, there is a continuous call in the team literature to further expand our understanding of dynamic team process not only by using survey methodology, but also by applying real-time behavior observation techniques $[3,18]$. With this paper, we want to address this call by introducing a new behavioral coding system for analyzing idea finding interactions (AIFI). 


\section{Background}

\subsection{The creative problem-solving process}

Much research on creativity in groups follows Osborne's well-known concept of creative problemsolving [30]. To structure the creative problem-solving process, Osborn [25] suggested several stages, which accrue to three overarching phases: fact-finding, ideafinding, and solution-finding. Fact-finding includes an analysis and definition of the problem. Team members are advised to pick out and identify the core problem, to point out the characteristics of the problem, and to gather and analyze data relating to a challenge at hand [10]. After having established a shared understanding of the main problem, the team transitions to the idea finding phase of creative problem-solving, which is the focus of the current research.

Osborn suggested that idea finding consist of two separate stages. First, team members are advised to generate many and manifold ideas (i.e. idea production). This includes coming up with tentative, and sometimes even wild, ideas. Second, team members need to further develop these ideas which can include adding to ideas, modifying ideas, and combining ideas (i.e. idea development). The goal is to pick the most promising ideas and proceed to the solution phase.

During solution finding, the team evaluates the tentative solution, for example, by testing and integrating user feedback and applying it in other contexts. Finally, the team adopts the final solution and continues with the implementation of an idea.

\subsection{Idea finding: More than brainstorming}

In order to generate a lot of ideas during the first stage of idea finding, Osborn [25] developed what has become his legacy, the brainstorming technique. To facilitate brainstorming, Osborn suggested several guidelines which include focusing on quantity of ideas, not criticizing or judging ideas, building on the ideas of others, and freewheeling which describes the spontaneous expression of (wild) ideas. Put differently, "it is easier to tame down than to think up" [25]. According to Osborn, the creative problemsolving process is not tied to a fixed schedule but can span across multiple meetings and iterations depending on the specific task.

Despite its initial appeal and being widely used in both research and practice, brainstorming has come under a lot of scrutiny. Studies even suggests that, in comparison to nominal groups, brainstorming groups perform worse in terms of idea quality and idea quantity [24]. Against this background, it seems to be only a small advantage that brainstorming groups tend to be more satisfied and pleased with the results they come up with [27]. Accordingly, practitioners who want to make use of the brainstorming technique might experience the dilemma that they are not able to offer their teams an effective and satisfying approach towards creating new ideas.

Such a perspective however neglects that brainstorming should be just the first step in the idea finding phase [25]. After team members brought their ideas to the table, they are tasked with further developing these ideas. This second step goes beyond mere brainstorming, as shown in Osborn's model of the creative problem-solving process. Unfortunately, the idea development stage is often given less attention, although developing ideas is inevitable in order to move forward in the creative problem-solving process. Previous research has shown that teams are not likely to have problems producing a lot of original, novel, and uncommon ideas [35]. Rather, it is critical to evaluate these ideas for their practicability, since they "may be unique or uncommon for good reason!" ([32], p. 92).

This distinction between idea production, which concerns the thinking up of ideas and can be aided by brainstorming, and idea development, as the selection and reprocessing of ideas, also aligns with Guilford's [7] concept of divergent (producing many ideas) and convergent (channeling efforts towards one solution) thinking. Following this paradigm, an ideal team broadens its thinking and focus to produce a wealth of ideas in the divergent phase, but then channels its thinking and focus to agree on a specific idea in the convergent phase [33]. Next to problems associated with divergent thinking or brainstorming, it can also be difficult for a team to stir successfully through the convergent stage of idea development [30].

\subsection{Understanding behavioral dynamics during the idea finding process}

We argue that more research is needed that zooms in on how teams develop specific ideas in order to examine idea finding, as the main part of creative problem-solving, more fully $[10,36]$. In other words, idea finding is more than just brainstorming. The goal of the new coding system we are about to introduce therefore lies on including both idea production and idea development to more fully reflect Osborn's original notions of idea finding and to also build on the literature of divergent and convergent thinking. In sum, we aim to introduce a theoretically-sound coding system that supports the efforts of creativity and innovation researchers. 


\section{The AIFI system}

\subsection{Goals of the AIFI system}

The AIFI system is aimed at analyzing the finegrained temporal interaction processes unfolding during creative problem-solving. The AIFI system sets out to capture the entire flow of conversational events exchanged among team members in creativity or innovation settings. As such, the behavioral codes of the AIFI system are meant to be exhaustive and mutually exclusive [1].

When developing the AIFI system, we wanted to create an efficient coding system that is easy to use and learn. At the same time, we wanted to arrive at a coding system that has high external validity and can be easily used for providing teams with feedback. In particular, and inspired by previous research on visualizing the temporal dynamics of change conversations [14], we wanted the results of the AIFI analysis to be easily visualized. The idea was to capture whether teams are currently moving towards new ideas (i.e. if ideas flow) or whether teams are stuck in the idea finding process (i.e. inertia momentum).

\subsection{Steps in developing the AIFI system}

The AIFI system was developed both inductively (i.e. bottom-up) and deductively (i.e. top-down). In doing so, we followed existing guidelines for developing a new behavioral coding system $[1,41]$. During the inductive phase, we observed video recorded idea-generation teams to an initial understanding of the team dynamics at play during innovative tasks, and to decide on a unitizing rule to segment the communication flow. For the deductive phase, we aimed to systematize our observations by building and expanding on existing interaction coding systems.

3.2.1. Pilot data for developing the AIFI system inductively. As part of our inductive approach, we conducted a detailed task analysis. Initially, the Idea finding interactions of twelve student teams participating in a Design Thinking workshop were video-recorded. All workshops were conducted by facilitators with a professional background in Design Thinking and lasted for three days. Ad-hoc teams of 4 to 5 students were taught the basics of Design Thinking, and they applied their new knowledge to tasks of project partners. The 12 teams consisted of a total of 53 team members. On average, team members were 25.50 years old $(S D=1.50)$, and most participants were male $(79.2 \%$ male).

On the second day of the workshops, the teams were asked to generate ideas for their given challenges. These team interaction episodes were videotaped and discussed among the authors of the current paper as well as student assistants. Later, videos were annotated using Interact software [22], and we decided on a unitizing rule for sequencing the team interaction process into individual behavioral units.

\subsubsection{Literature research for developing the AIFI} system deductively. To relate our observations to existing coding systems, we carried out a thorough literature search covering previous literature and empirical studies on team creativity and team innovation [5, 27, 29, 42] as well as on team interaction coding [18, 44]. We consolidated both universal group interaction coding systems $[2,6,13$, $34]$ as well as more setting-specific coding systems for idea generation $[11,15,37]$. For instance, the code "blocking" was adapted from the Interaction Dynamics Notation system [37] and relates to our observation that some team members undermine the creative process.

\subsection{Unitizing}

In line with previous research on coding group interactions $[2,13]$, the unit of analysis is a thought unit. A thought unit is the smallest meaningful segment of behavior that constitutes an entire thought. In practice, this is often a sentence or statement. However, it can also be a single word ("Okay") or an incomplete sentence (e.g. when a team member calls out a single idea during a brainstorming episode). Moreover, a new behavioral event is parsed whenever the main argument changes (e.g. several ideas are voiced in a row) or when a team member states several statements in a row (e.g. first stating a new idea and then explaining an idea).

Unitizing is carried out in a sequential fashion, meaning the AIFI systems leads to a continuous stream of coded behavior. This way, the patterns of Idea finding over time can be analyzed.

\subsection{Description of the AIFI system}

An overview of the AIFI system is provided in Table 1. The AIFI system categorizes behaviors according to their function for the group discussion.

This means, that the AIFI systems does not provide a qualitative content-analysis of the creative team process but a quantitative analysis in terms of behavior 
counts. In line with our overall goal to develop a sound coding system that is both suitable for research and practice, we decided on a hierarchical structure.

Table 1: Overview of the AIFI system

\begin{tabular}{|c|c|c|}
\hline $\begin{array}{l}\text { Emergent } \\
\text { state }\end{array}$ & Process & $\begin{array}{l}\text { Behavioral } \\
\text { codes }\end{array}$ \\
\hline \multirow{6}{*}{$\begin{array}{l}\text { Group flow } \\
\qquad(+1)\end{array}$} & \multirow{4}{*}{ Idea facilitation } & Idea expression \\
\hline & & Idea explanation \\
\hline & & $\begin{array}{c}\text { Idea } \\
\text { development }\end{array}$ \\
\hline & & Knowledge \\
\hline & \multirow{2}{*}{$\begin{array}{c}\text { Team spirit } \\
\text { facilitation }\end{array}$} & Support \\
\hline & & Humor \\
\hline \multirow{3}{*}{\multicolumn{2}{|c|}{ Neutral (0) }} & $\begin{array}{c}\text { Process } \\
\text { organization }\end{array}$ \\
\hline & & $\begin{array}{c}\text { Simultaneous } \\
\text { talk }\end{array}$ \\
\hline & & Other \\
\hline \multirow{6}{*}{$\begin{array}{c}\text { Inertia } \\
\text { momentum } \\
(-1)\end{array}$} & \multirow{4}{*}{ Idea inhibition } & $\begin{array}{c}\text { Off-topic } \\
\text { conversation }\end{array}$ \\
\hline & & $\begin{array}{l}\text { Loss in detail and } \\
\text { repetition }\end{array}$ \\
\hline & & Silence \\
\hline & & Blocking \\
\hline & \multirow{2}{*}{$\begin{array}{l}\text { Team spirit } \\
\text { inhibition }\end{array}$} & $\begin{array}{l}\text { Relationship } \\
\text { conflict }\end{array}$ \\
\hline & & Complaining \\
\hline
\end{tabular}

On the most coarse-grained level, the AIFI system differentiates two emergent team states: group flow versus inertia momentum. The group flow category describes that the team is currently moving in the direction of generating and developing new ideas. Thus, all behaviors that fall in this category are rated +1 to indicate that they help towards generating an idea. On the contrary, inertia momentum describes that team members are moving away from finding new ideas and are rated -1 . Behaviors that we expect to neither support nor hinder the finding of ideas are coded as neutral behavior and receive a rating of zero. For practitioners who are interested to use a (live) coding system that is easy to apply but can still trace the moment-to-moment dynamics, this might be the level to focus on. We provide a suggestion for how to visualize this dichotomy (i.e. idea flow versus inertia momentum) in the discussion.

At the second level, the AIFI system differentiates five behavioral team process categories: idea facilitation versus idea inhibition, team spirit facilitation versus team spirit inhibition, and again neutral behaviors (which are not yet further subdivided at this point in the coding process).
Zooming in even further, the AIFI system differentiates 15 fine-grained behavioral categories.

3.4.1. Codes facilitating Idea finding (+1). Idea expression: This code is used when a team member comes up with a new idea but does not further elaborate on the idea. Such statements are typically very short ("How about using a different color scheme?").

Idea explanation: When team members do not just state an idea but briefly explain or describe an idea, this is coded as idea explanation ("Red is much more eye-catching").

Idea development: This code is applied when an idea that has been mentioned before is further developed by modifying, combining, comparing, or prioritizing the idea [5]. Thus, new perspectives are added to an existing idea ("How about adding a flashing indicator?").

Knowledge: When team members contribute relevant (domain-specific) knowledge ("Distinguishing between red and green is more intuitively because you know it from traffic lights") or reference personal experience (e.g. "When I was in school...") that moves the idea finding process forward, this is coded as knowledge. Providing knowledge to a team discussion can often serve as the basis to come up with new ideas and concepts [43].

3.4.2. Codes facilitating team spirit $(+1)$. Support: When team members express their explicit agreement or appreciation with an idea, person, or the process, this is coded as support (e.g. "That's a great idea!", "How cool", "I like that a lot"). Requesting an idea to be further explained (e.g. "Could you give an example?") or asking for opinions is also coded as support ("What do you think?")

Humor: Humorous remarks, jokes, and laughter are coded as humor. If team members laugh at someone's expense this is coded as relationship conflict (see below).

3.4.3. Neutral codes (0). Process organization: References to time (e.g. "We only have 5 minutes left"), reading out task descriptions, mentioning the overall task, or asking how to proceed (e.g. "Who wants to go first?") is coded as process organization.

Simultaneous talk: Even though coders are encouraged to identify the speaker currently heading the conversation, sometimes two or more team members talk at the same time. The code simultaneous talk is used to capture these events.

Other: Statements that do fit not into any of the functional categories are coded as other behavior in order to make the coding exhaustive. 


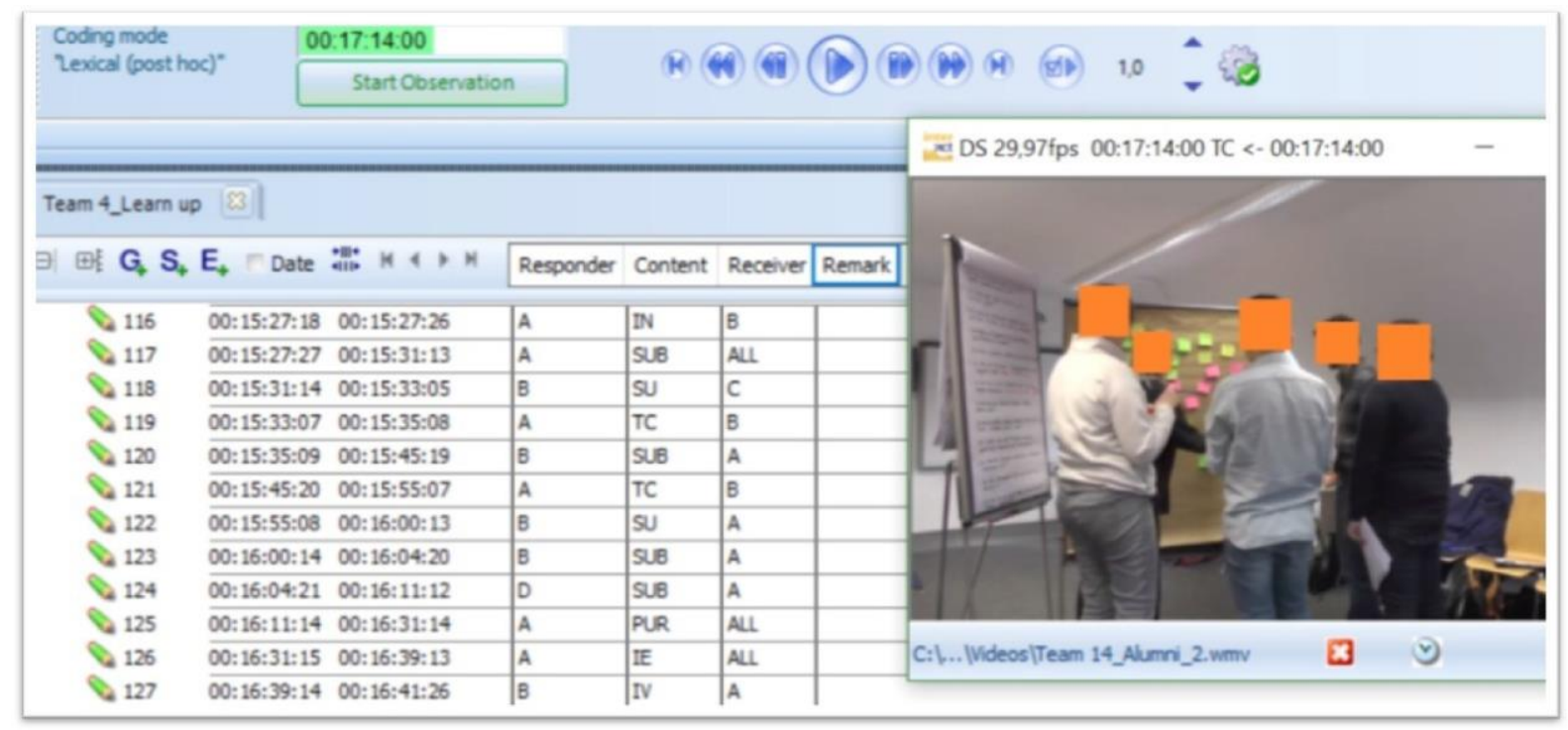

Figure 1: Stream of idea finding interaction coded with the AIFI system using Interact software [22]

3.4.4. Codes inhibiting idea finding (-1). Off-topic conversation: Statements not contributing to idea finding and that signal a lack of interest are coded as off-topic conversation (e.g. "Did you watch the game last night?", "What could we have for lunch today?").

Loss in detail and repetition: Very long explanations that do not provide new information are coded as loss in detail and repetition. The code is also used, if team members keep referring to their previous ideas. This repetitive behavior is often shown, when a team member cannot let go of his or her idea although the other group members show no interest progressing with this idea.

Silence: Interactions during idea finding are typically fast-paced and energetic. If team members do not say anything for more than 6 seconds, the code silence is used.

Blocking: The code blocking is used when team members disagree with other team members or their ideas or refer to negative feelings ("I hate this idea").

\subsubsection{Codes inhibiting team spirit (-1). Relationship} conflict: If team members show aggression, personally attack somebody else, make subliminal jokes at the expense of another team member, or try to undercut the authority or competence of a fellow team member (e.g. "You are not more than a student"), this is coded as relationship conflict.

Complaining: When team members express disinterest or pessimism ("This sucks"), try to find a scapegoat ("This is all XY fault"), try to end the discussion early ("Can we stop already?"), this is coded as complaining.

\subsection{Technical requirements}

Coding with the AIFI system does not have to be software assisted. Nevertheless, we recommend a software solution to minimize the coding effort and to simplify data processing and analysis. We used Interact software in our research [22]. A screenshot illustrating how the annotation looks like using Interact software is provided in Figure 1.

\section{A first test of the AIFI system}

\subsection{Participants and procedure}

Participants were 80 psychology students who received course credit for their participation in an idea finding experiment. In total 20 teams, each consisting of four team members, worked on two tasks. One challenge was to help the university becoming more sustainable and environmentally friendly. The other challenge was to create ideas concerning how the city can become more appealing for its residents and tourists. Each team worked on both challenges, one time with a facilitator and one time without a facilitator. Accordingly, the sample consists of 40 idea finding sessions. Participants were on average 23.30 years old $(S D=5.18)$, and $77.5 \%$ of the participant were female which is representative for psychology students.

Each idea finding session took 30 minutes and consisted of a divergent phase (idea generation; 15 minutes), a convergent phase (idea selection; 10 minutes), and a final elaboration phase (five minutes). 
During the elaboration phase, participants were asked to specify their final idea and prepare a flipchart. Each team was provided with a time-timer clock to support their time management. Moreover, a student assistant ensured that each team kept up with the indicated time. The final idea was presented by one team member using the flipchart. These presentations were no longer than one minute and served to later asses the ideas by external raters. All team interactions and the final presentations were videotaped.

\subsection{Variables}

4.2.1. Idea quantity. In order to assess idea quantity, we asked two student assistants to count all post-its that were produced by the teams during the divergent phase. To assess the level of agreement among both raters, we calculated a two-way random intraclass correlation (ICC) with absolute agreement (average measure). There was nearly perfect agreement between both observers $(I C C=.96)$ so that both scores were averaged into one score.

4.2.2. Idea quality. In order to rate the quality of an idea, we developed a new measure that captures both the feasibility of an idea (three items, e.g. "Given the technical resources, the implementation of the idea is possible"; ICC $=.62$ ) and its user-centeredness (four items, e.g. "The idea solves problems that people encounter in their daily routines"; $I C C=.38$ ). Raters were three staff members of the university's campus innovation team. Their mission is to spot promising ideas to improve student life at the campus and help teams realize their projects by providing expertise and resources.

4.2.3. Perceived effectiveness. To include a measure that also takes into account the team members' own experience and evaluation, we assessed their perceived effectiveness with the idea finding process. We used a scale by Lemieux-Charles and colleagues [19] (e.g. "My team's overall performance met my expectations"). Items were answered on a five-point Likert scale $(1=$ strongly disagree; 5 = strongly agree). Reliability for this scale was satisfactory (Cronbach's alpha $(\alpha)=.82$ without facilitator and Cronbach's alpha $\alpha=.83$ for sessions with a facilitator).

4.2.4. Behavioral coding (AIFI system). Coders were two student assistants familiar with using Interact software who received training in using the AIFI system. Specifically, they participated in a joined coding workshops. The unitizing rules as well as the definitions and examples of the different coding categories were explained and discussed.

Interaction coding was carried out as described above. Overall, we coded a total of 21.435 behavioral events, which means that on average, we observed 714 events per 30-minute idea finding session. To ensure adequate reliability of the AIFI system, seven videos were double-coded to assess the point-by-point agreement between both coders. Specifically, using Interaction software, an alignment Kappa coefficient was calculated that accounts for the fact two observers cannot parse each behavioral event at the exact same second. We specified two behavioral events to be a match/mismatch if they overlapped at least $50 \%$. Moreover, we specified non overlapping events to be a match/mismatch if their onset times were within a tolerance window of 1 second.

Kappa values can range from -1 to 1 , with higher values indicating higher agreement. We followed conventional cutoff criteria as proposed by Landis and Koch [17]: .21-.40 = fair, .41-.60= moderate, $.61-.80$ $=$ substantial, and $.81-1.00=$ almost perfect.

\subsection{Results}

Means, standard deviations, and correlations between all study variables are shown in Table 2. To visualize the temporal flow of the coded team interaction, we plotted the coded data for one sample team (see Figure 2).

4.3.1. Reliability. Interrater reliability for the AIFI's process codes (i.e. $2^{\text {nd }}$ level) yielded a Kappa value of .53 indicating moderate agreement among both coders. Interrater reliability for the more fine-grained 15 individual codes (i.e. $3^{\text {rd }}$ level) was .44 , hence still in the moderate range. Interestingly, we found that our first coder followed a more detailed unitizing approach and identified more behavioral events than the second coder. Future coder training should place higher emphasis on how to sequence the behavior stream into behavioral units.

4.3.2. Validity. In line with our expectations, idea facilitating behaviors were associated with perceived effectiveness $(r=.34 ; p=.03)$ and a higher number of generated ideas $(r=.31 ; p=.05)$. Contrary to what we would expect, teams that produced more ideas, perceived themselves as less effective $(r=-.34 ; p=$ $.03)$. Findings also revealed that team spirit facilitating interactions such as support and humor were related team members' perceived effectiveness $(r=.56$; $p<$ $.001)$. 
Table 2: Means, standard deviations, and intercorrelations between study variables

\begin{tabular}{|l|r|r|r|r|r|r|r|r|c|}
\hline & Mean & \multicolumn{1}{|c|}{ SD } & $\mathbf{1}$ & $\mathbf{2}$ & $\mathbf{3}$ & $\mathbf{4}$ & $\mathbf{5}$ & $\mathbf{6}$ & $\mathbf{7}$ \\
\hline 1. User-centeredness & 5.70 & .57 & & & & & & & \\
\hline 2. Feasibility & 4.60 & .67 & $-.31^{\dagger}$ & & & & & & \\
\hline 3. Idea Quantity & 24.28 & 7.11 & $.27^{\dagger}$ & -.17 & & & & & \\
\hline 4. Perceived Effectiveness & 3.95 & .44 & -.19 & -.01 & $-.34^{*}$ & & & & \\
\hline 5. Idea Facilitating (AIFI) & 153.93 & 28.30 & .11 & .03 & $.31^{\dagger}$ & $.34^{*}$ & & & \\
\hline 6. Team Spirit Facilitating (AIFI) & 199.50 & 51.83 & .09 & -.19 & -.07 & $.56^{* *}$ & $.54^{* *}$ & & \\
\hline 7. Idea Inhibition (AIFI) & 57.50 & 15.40 & .16 & .05 & .26 & -.13 & $.28^{\dagger}$ & .23 & \\
\hline 8. Team Spirit Inhibition (AIFI) & 1.40 & 1.39 & -.20 & -.19 & -.09 & .08 & -.01 & .14 & .22 \\
\hline
\end{tabular}

$N=40$ teams. ${ }^{*} p<.10 ; * p<.05 ; * * p<.01$

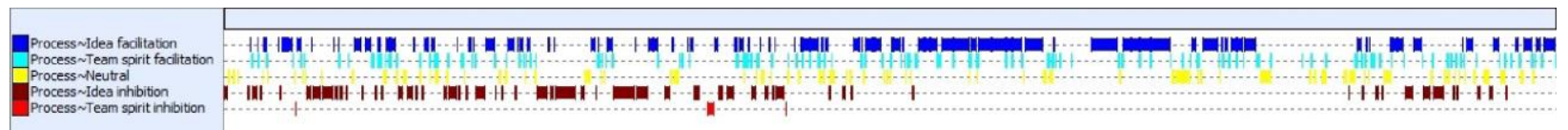

Figure 2: Time line chart of a 30-minute idea finding episode coded with the AIFI system

Moreover, idea facilitating and team spirit facilitating behaviors were correlated $(r=.54 ; p<$ $.001)$, suggesting that they complement one another. Unexpectedly, idea facilitation and idea inhibitive behaviors were also correlated $(r=.28 ; p=.08)$.

Another unexpected finding, which is not the focus of the current study but worth mentioning, is the fact that feasibility and user-centeredness tend to contradict one another $(r=-.31 ; p=.05)$. This finding suggests that realistic and feasible ideas are not the ideas that meet users' needs. Furthermore, the number of ideas generated in the idea generation phase seems to indicate that the team will produce an idea that is rated as user-centered $(r=.27 ; p=.094)$. This supports Osborn's [25] claim that during brainstorming, a team should create a high number of ideas.

\section{Discussion}

More than seven decades ago, Osborn [25] articulated the timeless need to advance the productivity of groups. He developed a creative problem-solving process, with the idea finding phase as the backbone of this process. However, previous research questioned the effectiveness of the idea finding phase, either due to problems concerning idea generation [4] or idea evaluation [30].

Despite these problems, however, teams and organizations are still required to continuously create new solutions for emerging challenges. Thus, it is not expected that idea finding teams will vanish from the organizational context within the near future.

The aim of this paper was to enhance our understanding of the fine-grained micro-dynamics unfolding in creativity teams during the idea finding phase. We thereby contribute towards recent calls to study team members' interaction process more closely [10]. It does not suffice putting together a group of highly creative individuals because team members' potential to come up with ideas can be offset when dysfunctional group dynamics emerge that undermine finding new and feasible ideas [39]. We introduced the AIFI system, a coding instrument for capturing the moment-to-moment dynamics of creative team interactions to better understand which specific team behaviors characterize successful idea finding. Findings from an experimental study provided initial support for the criterion-related validity of the AIFI coding system. In particular, findings disclosed that team members perceive themselves as more effective when they showed more idea facilitation (e.g. explaining ideas) and team spirit facilitation (e.g. humor) behaviors. In addition, our analyses also revealed some unexpected associations such as a correlation between idea facilitation behaviors and idea inhibition (e.g. blocking) behaviors. One explanation for this finding might be that those teams who express and develop more ideas also have more opportunities to block and criticize these ideas.

\subsection{Theoretical implications}

Thus far, stirring through the idea finding phase has been a bit like cooking without knowing the exact recipe. There are some ingredients (e.g. go for quantity or defer judgement) that everyone would intuitively agree on. However, knowledge about the exact amount of these ingredients was missing. So far, we had no reliable measurement instrument to quantify these ingredients and understand how they interact to form 
a delicious outcome. Thus, to be able to replicate a 'good' recipe, this paper presented the AIFI system.

First, we examined natural occurring interactions of innovation teams. The behavioral codes derived from this first step have thus a high external validity. Second, we built on previous research on group interaction coding schemes to systemize our observations and anchor the AIFI system in a diverse and solid research base. We aimed to develop a tool that allows to disclose temporal interaction processes and emergent behavioral patterns, which in turn can contribute to the theoretical framework that is currently available for team process researchers. A first application of the AIFI system revealed that behaviors supporting an idea are more frequently shown in teams that also show more behaviors supporting the team spirit. Using computer software, we were further able to map the temporal flow of idea finding interactions (see Figure 2). Such visualizations can yield additional insights and improve our understanding of how team progress in finding new ideas. Interpreting Figure 2, findings suggest that idea inhibitive behaviors are more frequent in the divergent phase (the first half, minutes 1-15) than in the convergent phase (the second half, minute 16-25). Finding sound explanations and testable hypotheses for this finding could help to broaden the theoretical basis of group creativity research.

\subsection{Practical implications}

The AIFI system is thought to be applicable across a wider range of group creativity settings, including traditional brainstorming sessions, group creativity interactions during organizational meetings, and team innovation tasks more generally. One requirement is that team interaction is focused on generating new ideas and solutions rather than on sharing information only. The AIFI system can be applied to team data collected in the laboratory and the field. A version for real-time (ad-hoc) coding is currently under development. The goal of the ad-hoc coding system is that facilitators do not have to interrupt a team but can still provide immediate feedback [28]. That is, a stream of coded events could be displayed on a wall in the background of the working space and provide visual feedback about a team's dynamic in real-time [14]. To make this tool intuitively to use, we suggest building on the dichotomy of group flow versus inertia momentum to indicate whether a team moves away or towards a new idea. Distinguishing only between three codes $(+1,0,-1)$ should be suitable for ad hoc (realtime) coding.

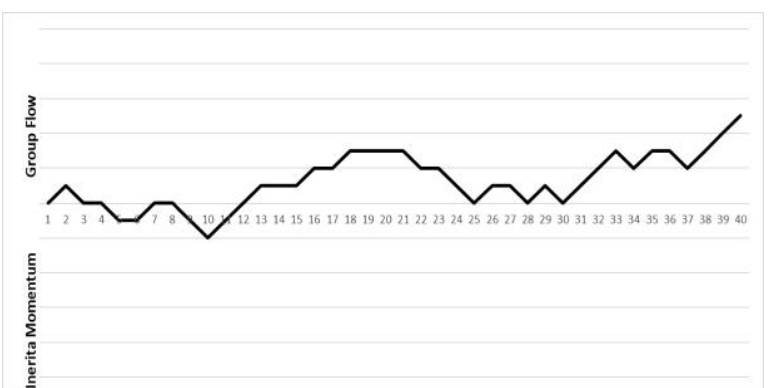

Figure 2. Illustration of the dynamics in an idea finding team (adapted from [14], p. 423). Events that help to progress an idea contribute towards the group flow and are scored (+1), events that hinder the process to find an idea and contribute towards the inertia momentum and are scored $(-1)$. Events that are neither idea supportive, nor inhibitive are scored as neutral (0).

Figure 2 shows a hypothetical team interaction episode, consisting of 40 coded events. For this illustration, we assume that the first half (events 1-20) depicts the divergent thinking phase and the second half (events 21-40) the convergent thinking phase.

At the beginning (events 1-10), the team started with idea inhibitive behaviors, such as blocking further idea development. However, in the second half of the idea generation session, the team starts to come up with ideas and team members tend to build on each other's ideas, which is indicated by an increase in the curve during events 11-20. Similar to the divergent thinking phase, the convergent thinking started with idea and team spirit inhibitive behaviors, which lowered the curve (events 21-30). As team members might have used this visual feedback about their dysfunctional behavior, they switched their working mode and showed more behavior indicating that the group flows towards a good solution (events 31-40).

Given this illustration, it becomes clear that the development of such a group support system moves forward the innovation management process. Such system would provide immediate feedback to the team, without interrupting its process. Using the AIFI system in this way could help a team make use of its creative potential more fully. In this regard, the role of a facilitator is similar to that of a good teacher meaning that he or she should only intervene when the team gets off track [20].

\subsection{Future research}

5.3.1. Distinguish between divergent and convergent thinking stages. Since the idea finding phase consists of two distinct stages, it is important to understand whether one could examine significant differences between these thinking modes [10]. Thus, 
using the AIFI system to examine behavioral dynamics and how these patterns might differ according to the idea generation and idea evaluation stage could further enlighten the understanding of beneficial group interactions.

5.3.2. Sequence analysis. Thus far, we only used the AIFI system to count behaviors. Another possible application of the system would be to use it for sequence analysis. Using sequence analysis allows analyzing which behavior by one team member increases the likelihood of another team member's behavior [1]. For instance, previous research [31] has shown that ideas are often voiced after a short pause rather than after periods of increased talk. This might also be a promising avenue for facilitators who could align their communication behavior accordingly and support the idea finding process.

5.3.4 Refining the AIFI. Although the AIFI was successfully applied to 40 idea finding sessions, results of the application suggest room for improvement. First, the interrater reliability is only moderate, suggesting that more coder training is necessary to achieve higher agreement among coders. Other than that, one might also think about refining the coding system. For instance, the codes "idea explanation" and "idea development" have conceptual overlap and it might be hard to distinguish between these codes. A possible solution might be to merge these two codes into one.

Another aspect that needs refining is our measure of the quality of an idea. Especially the intraclass correlation between raters regarding the usercenteredness of an idea is rather week. This is problematic since it means that much of the variance is not explained by our predictors (i.e. the AIFI codes). Accordingly, one might re-analyze the ideas that were video-taped using more advanced measures that assess the quality of an idea more fully.

\section{Conclusion}

By presenting a coding system that allows to analyze a team's interaction during idea finding, this paper enhanced our understanding regarding intragroup micro-dynamics that foster or undermine creativity and innovation in teams. By its hierarchal structure, the AIFI system is easy to apply for practitioners and also allows researchers to carry out a fine-grained analyses of team processes. Given this flexible applicability, future research might refine and advance the AIFI to increase its reliability and validity.

\section{References}

[1] Bakeman R. and V. Quera, Sequential Analysis and Observational Methods for the Behavioral Sciences, Cambridge University Press, Cambridge, MA, 2011.

[2] Bales, R.F., Interaction Process Analysis, AddisonWesley Press, Cambridge, MA, 1950.

[3] R.F. Baumeister, K.D. Vohs, and D.C. Funder, "Psychology as the Science of Self-Reports and Finger Movements: Whatever Happened to Actual Behavior?", Perspectives on Psychological Science, 2(4), 2007, pp. 396403.

[4] M. Diehl and W. Stroebe, "Productivity Loss in Brainstorming Groups: Toward the Solution of a Riddle", Journal of Personality and Social Psychology, 53(3), 1987, pp. 497-509.

[5] Eberle, R.F., Scamper: Games for Imagination Development, D.O.K. Publishers, Buffalo, NY, 1971.

[6] G.C. Futoran, J.R. Kelly, and J.E. McGrath, "TEMPO: A Time-based System for Analysis of Group Interaction Process", Basic and Applied Social Psychology, 10(3), 1989, pp. 211-232.

[7] J.P. Guilford, "The Structure of Intellect", Psychological Bulletin, 53(4), 1956, pp. 267-293.

[8] S. Harvey, "Creative Synthesis: Exploring the Process of Extraordinary Group Creativity", Academy of Management Review, 39(3), 2014, pp. 324-343.

[9] B.A. Hennessey and T.M. Amabile, "Creativity", Annual Review of Psychology, 61, 2010, pp. 569-598.

[10] S.G. Isaksen and Treffinger, D.J., "Celebrating 50 Years of Reflective Practice: Versions of Creative Problem Solving", The Journal of Creative Behavior, 38(2), 2004, pp. 75-101.

[11] M.H. Jackson and M.S. Poole, "Idea-Generation in Naturally Occurring Contexts", Human Communication Research, 29(4), 2003, pp. 560-591.

[12] S. Kauffeld and N. Lehmann-Willenbrock, "Meetings Matter: Effects of Team Meetings on Team and Organizational Success", Small Group Research, 43(2), 2012, pp. 130-158.

[13] S. Kauffeld, N. Lehmann-Willenbrock, and A.L. \& Meinecke, "The Advanced Interaction Analysis for Teams (act4teams) Coding Scheme.", in The Cambridge Handbook of Group Interaction Analysis, E. Brauner, M. Boos, and M. Kolbe, Editors. Cambridge University Press: Cambridge, MA, 2018, pp. .422-431.

[14] F. Klonek, H.F.K. Paulsen, and S. Kauffeld, "They Meet, They Talk ... but Nothing Changes: Meetings as a Focal Context for Studying Change Processes in Organizations", in The Cambridge Handbook of Meeting Science, J.A. Allen, N. Lehmann-Willenbrock, and S.G. Rogelberg, Editors. Cambridge University Press.: Cambridge, MA, 2015, 413-439.

[15] C.-Y. Kou and S. Harvey, "Coding Scheme for Group Creativity", in The Cambridge Handbook of Group Interaction Analysis, E. Brauner, M. Boos, and M. Kolbe, 
Editors. Cambridge University Press: Cambridge, MA, 2018, 537-546.

[16] S.W.J. Kozlowski, "Advancing Research on Team Process Dynamics", Organizational Psychology Review, 5(4), 2015, pp. 270-299.

[17] J.R. Landis and G.G. Koch, "The Measurement of Observer Agreement for Categorical Data", Biometrics, 33(1), 1977, pp. 159-174.

[18] N Lehmann-Willenbrock and J.A. Allen, "Modeling Temporal Interaction Dynamics in Organizational Settings", Journal of Business and Psychology, 33, 2018, pp. 325-344.

[19] L. Lemieux-Charles, M. Murray, G. Ross Baker, J. Barnsley, K. Tasa, and S.A. Ibrahim, "The Effects of Quality Improvement Practices on Team Effectiveness: A Mediational Model", Journal of Organizational Behavior, 23(5), 2002, pp. 533-553.

[20] K. Lewin, "Cultural Reconstruction", in Resolving Social Conflicts: Selected papers on Group Dynamics, Lewin, G, W., Editor. Harper \& Row: New York, NY, 1948, pp. 34-42.

[21] Litchfield, R.C., "Brainstorming Reconsidered: A Goal-Based View", Academy of Management Review, 33(3), 2008, pp. 649-668.

[22] Mangold, Interact Coding Software, 2015.

[23] McGrath, J.E., Social Psychology: A Brief Introduction, Holt Rinehart and Winston, New York, NY, 1964.

[24] B. Mullen, C. Johnson, and E. Salas, "Productivity Loss in Brainstorming Groups: A Meta-Analytic Integration", Basic and Applied Social Psychology, 12(1), 1991, pp. 323.

[25] Osborn, A.F., Applied Imagination: Principles and Procedures of Creative Problem-solving., Scribner, New York, NY, 1953.

[26] S.B.F., Paletz, C.D. Schunn, and K.H. Kim, "Intragroup Conflict Under the Microscope: Micro-Conflicts in Naturalistic Team Discussions", Negotiation and Conflict Management Research, 4(4), 2011, pp. 314-351.

[27] P.B. Paulus, "Groups, Teams, and Creativity: The Creative Potential of Idea-generating Groups", Applied Psychology, 49(2), 2000, pp. 237-262.

[28] P.B. Paulus and M.T. Dzindolet, "Social Influence Processes in Group Brainstorming", Journal of Personality and Social Psychology, 64(4), 1993, pp. 575-586.

[29] R. Reiter-Palmon, T.d. Vreede, and de Vreede, G.J., "Leading Interdisciplinary Creative Teams: Challenges and Solutions", in Creativity and Leadership in Science, Technology, and Innovation, B.R. Martin, C.M. Allwood, M.D. Mumford, and S. Hemlin, Editors. 2013. Routledge: New York, NY, pp. 240-267.

[30] E.F. Rietzschel, B.A. Nijstad, and W. Stroebe, "The Selection of Creative Ideas after Individual Idea Generation: Choosing between Creativity and Impact", British Journal of Psychology 101, 2010, pp. 47-68.

[31] R.B. Ruback, J.M. Dabbs, and C.H. Hopper, "The Process of Brainstorming: An Analysis with Individual and
Group Vocal Parameters", Journal of Personality and Social Psychology, 47(3), 1984, pp. 558-567.

[32] M.A. Runco and G.J. Jaeger, "The Standard Definition of Creativity", Creativity Research Journal, 24(1), 2012, pp. 92-96.

[33] Sawyer, R.K., Group Genius: The Creative Power of Collaboration, Basic Books, New York, NY, 2007.

[34] C.C. Schermuly and W. Scholl, "The Discussion Coding System (DCS)—A New Instrument for Analyzing Communication Processes", Communication Methods and Measures, 6(1), 2012, pp. 12-40.

[35] I. Seeber, G.-J. de Vreede, R. Maier, and B. Weber, "Beyond Brainstorming: Exploring Convergence in Teams", Journal of Management Information Systems, 34(4), 2017, pp. 939-969.

[36] I. Seeber, I., R. Maier, P. Ceravolo, and F. Frati, "Tracing the Development of Ideas in Distributed, ITSupported Teams During Synchronous Collaboration", in ECIS 2014 proceedings: 22th European Conference on Information Systems ; Tel Aviv, Israel, June 9-11, 2014, M. Avital, J.M. Leimeister, and U. Schultze, Editors. 2014: AIS Electronic Library.

[37] N. Sonalkar, A. Mabogunje, and L. Leifer, "Developing a Visual Representation to Characterize Moment-to-moment Concept Generation in Design Teams", International Journal of Design Creativity and Innovation, 1(2), 2013, pp. 93-108.

[38] S. Taggar, "Individual Creativity and Group Ability to Utilize Individual Creative Resources: A Multilevel Model", Academy of Management Journal, 45(2), 2002, pp. 315330.

[39] K. Tasa, S. Taggar, and G.H. Seijts, "The Development of Collective Efficacy in Teams: A Multilevel and Longitudinal Perspective", Journal of Applied Psychology, 92(1), 2007, pp. 17-27.

[40] A. Taylor and H.R. Greve, "Superman or the Fantastic Four? Knowledge Combination and Experience in Innovative Teams", Academy of Management Journal, 49(4), 2006, pp. 723-740.

[41] F. Tschan, J. Zimmermann, and N. \& Semmer, "Rules for Coding Scheme Development", in The Cambridge Handbook of Group Interaction Analysis, E. Brauner, M. Boos, and M. Kolbe, Editors. Cambridge University Press: Cambridge, MA, 2018, 191-207.

[42] D. van Knippenberg, "Team Innovation", Annual Review of Organizational Psychology and Organizational Behavior, 4(1), 2017, pp. 211-233.

[43] T.d Vreede, I. Boughzala, G.-J. de Vreede, and R. Reiter-Palmon, "The Team Creativity Model: An Exploratory Case Study", Journal of the Midwest Association for Information Systems, 2017(1), 2017, pp. 19-34.

[44] M.J. Waller and S.A. Kaplan, "Systematic Behavioral Observation for Emergent Team Phenomena", Organizational Research Methods, 21(2), 2016, pp. 500515. 